\title{
Crowdsourcing New Product Design on the Web: An Analysis of Online Designer Platform Service
}

\author{
Xin Dai, ${ }^{1}$ Pui-Sze Chow, ${ }^{2}$ Jin-Hui Zheng, ${ }^{2}$ and Chun-Hung Chiu' \\ ${ }^{1}$ Sun Yat-Sen Business School, Sun Yat-Sen University, 135 West Xingang Road, Guangzhou 510275, China \\ ${ }^{2}$ Institute of Textiles and Clothing, The Hong Kong Polytechnic University, Kowloon, Hong Kong \\ Correspondence should be addressed to Pui-Sze Chow; lpschow@outlook.com
}

Received 2 August 2013; Revised 7 October 2013; Accepted 8 October 2013

Academic Editor: Kannan Govindan

Copyright (c) 2013 Xin Dai et al. This is an open access article distributed under the Creative Commons Attribution License, which permits unrestricted use, distribution, and reproduction in any medium, provided the original work is properly cited.

\begin{abstract}
A designer is a core resource in the fashion industry. Successful designers need to be creative and quick to understand the business and wider environment in which they are operating. The Designer Platform Service (DPS), which combines the mechanism of crowdsourcing and group buying on the web, provides a platform for entrant designers to try their abilities in the real market practice. Freelance designers post design samples or sketches of products on the website of DPS, and consumers may preorder the products (each at a fixed price) online based on the design information. Once the number of ordering reaches or passes a certain threshold, that is, the minimum production quantity (MPQ), DPS will arrange for production and delivery according to the orders received. This novel service boosts the growth of entrant designers and links designing works with real markets directly. We are interested in how the price and MPQ decisions are made in DPS, with consideration of the entrant designer's objective, decision sequences, and customer demand structures. We develop Stackelberg games to model and derive the equilibrium solutions under individual scenarios. Our findings suggest feasibility of the DPS business model.
\end{abstract}

\section{Introduction}

Fashion is among the most important creative industries that enjoy a great deal of attention these days. Fashion designers are the core of value creation in the fashion industry, as they are normally the source of creativity. In the UK alone, the designer sector produced $£ 700$ million income in 2003 , of which $£ 75$ million (11 percent) was from license fees to designers [1]. The fashion industry is also a highly competitive industry, where product life cycles are short, economies gained by product differentiation are built on brand image, and product styling can be quickly imitated [2]. Designers have to play a dual role as creative individuals and as entrepreneurs in any provided complicated situation.

Entrant designers are an important source of innovation in the fashion industry. Yet, the professional lives of fashion entrant designers are particularly hard. It takes time and resources for advertising and promotion so that a new designer label can sustain a share in the market, whereas most new start-up designers are lacking those resources [3].
However, in the market practice, resources are often allocated on those established designers, as the market has proved the attractiveness of their products, so that the resources allocated could be expected with higher and less risky return. Without advertising and promotion, most entrant designers would start their career with a relatively low and uncertain demand for their design, until they build up their name and cultivate a market. The low and uncertain demand faced by entrant designers hinders their design from being carried to production, as this demand may be far lower than the minimum production quantity which is commonly applied in textile manufacturing. Even if the production can be made, the small production quantity and uncertain demand would lead to high production and inventory cost.

In the past decade, the internet has bred a new approach called "crowdsourcing" that help individuals make their innovative ideas feasible. Named by Howe [4], crowdsourcing refers to the method that solicits contributions from a large group of people from an online community in order to make a certain service, idea or product available or feasible. Howe [4] 
also defined the sponsoring crowd as "the new pool of cheap labor: everyday people using their spare cycles to create content, solve problems, and even do corporate R\&D.” Many works have been done recently, on crowdsourcing contests [5] and noncompetitive crowdsourcing ideation initiatives [6-8]. For example, the crowdsourcing t-shirt platform, teespring.com, provides a way for people to create and sell tshirts online to raise money. Here is how it works: first, people design a t-shirt and post it on the website, choose a goal, and set a price to launch a campaign page; second, people send the campaign page to supporters and let them preorder the $t$ shirts towards the campaign goal (preordering is free: buyers will only be charged if the goal is reached); third, people can continue to sell shirts past the goal until the campaign ends. Once it does, the platform handle production and shipment; people will get a check for the profit. The business model of teespring.com, which combines the mechanism of crowdsourcing and group buying on the web, has provided a platform for entrant designers to try their abilities in the real market practice. In this business model, there are two key decisions to make, the price of the product and the target volume for production (equal to the campaign goal in the case of teespring.com), which are similar to the widely discussed problem of "joint pricing-production decisions" in operation management $[9,10]$.

To better illustrate how this business model works, we consider a simplified case, Designer Platform Service (DPS), which works as a retailer connecting designers with customers and designers with manufacturers. Similarly, DPS crowdsources the product design tasks to the entrant designer community and allows the customers to make purchases in the way of group buying. First, entrant designers post design samples or sketches of products on the website of DPS, and then consumers preorder the product (each at a fixed price) online based on the product information. The entrant designer decides the selling price of the product, while the DPS decides the minimum production quantity (MPQ). Only when the preorder quantity reaches or passes the MPQ threshold will DPS arrange for production and delivery. Preorder customers then make their payment and get the products delivered. Otherwise, no production will be arranged, and no one will be charged. This novel service provides variety to customers, boosts the growth of entrant designers, and links designing works with real markets. In the whole process, we are most interested in how the price and MPQ decisions are made within DPS, with consideration of entrant designer's objective, decision sequences, and customer demand structures.

Through the study in the form of Stackelberg games and by considering various scenarios, we investigate the decisions in the mechanism with B2C transactions: an entrant designer utilizes DPS to promote her design to consumer, and we reveal the price-discovery mechanism in this e-market. We consider that in our model, the two key players, the entrant designer and DSP, have very different objectives. Due to the special stage of an entrant designer in her professional career, her priority mission is to leverage the creativity with market acceptance, so that she can later find out a way to boost her design to profitability. Our findings provide insights on how the mechanism protects DPS in pricing decision in a highly versatile and risky market, and how the mechanism provides a feasible path for entrant designers to test and adjust to the market with limited loss. Moreover, our findings suggest feasibility of the DPS business model.

The paper contributes in providing insights of equilibrium decisions in the combined business model of crowdsourcing and group buying. The paper is new in investigating a creative business model which could significantly utilize creativity and reduce cost. The paper is also innovative in considering business players having nonmonetary objectives. By investigating the two key decisions in the DSP, we show how an online platform could raise a business by crowdsourcing designs, arranging manufacturing and selling to group buying customers. Specifically, we derive optimal decisions for the two parties under the different objectives of the entrant designer, decision sequences, and market demands, through which we show how an entrant designer could benefit from DSP. The findings provide guidelines to practitioners in platform service and designers and help utilize creation with profit.

The remainder of the paper is organized as follows. In Section 2, we review the related literature. We construct the Stackelberg game models in Section 3 and find the best response decisions in Section 4. In Section 5, we derive the equilibrium decisions of the games with specific demand functions. We discuss and conclude in Section 6 with limitations and future research directions.

\section{Literature Review}

This paper relates to the literature (e.g., [11-13]) in service platform and group buying business on the web. Web-based group buying mechanisms are being widely used in both business-to-business (B2B) and business-to-consumer (B2C) transactions. $\mathrm{Li}$ and Lee [12] show that with the uncertain peer-produced services quality, a monopolistic platform provider has no incentive in offering multiple quality classes of service, while two competing platform providers may offer identical service contracts but still receive nonnegative profit. Anand and Aron [14] studied a monopolist offering Webbased group buying under different kinds of demand uncertainty. They derive the monopolist's optimal group-buying schedule under varying conditions of heterogeneity in the demand regimes and compare its profits with those obtained under the more conventional posted-price mechanism.

Pricing decision and determination of the minimum production quantity with price-dependent demand are the main focus of the models explored in this paper. In marketing and operations management literature, there is a huge amount of related studies under various settings and we review some of them as follows. For instance, Pasternack [15] studies the pricing decision and return policy for perishable products. Cai et al. [16] explore from the game-theoretic perspective the pricing scheme of a supply chain with dual channels that compete with one another. The authors show that a simple price discount contract can achieve channel coordination. In particular, the pricing issue has aroused the attention of many 
researchers when the demand is price-dependent. Petruzzi and Dada [17] investigate the optimal selling price and stocking quantity under the newsvendor domain with pricedependent demand. Yang et al. [18] consider an inventory system for noninstantaneous deteriorating items with pricedependent demand and develop an algorithm to solve the corresponding optimal price and order quantity when partial backlog is allowed. Chen et al. [19] suggest some coordination schemes for a supply chain with lead time consideration and price-dependent demand. Chiu et al. [20] suggest a mechanism for coordinating the pricing and stocking decisions for a supply chain with price-dependent demand.

To make use of the economy of scale, it is common for suppliers to impose some minimum quantity requirement on orders. Such practice is prevalent in the apparel industry, and Fisher and Raman [21] provide a well-studied example of minimum order quantity imposition in the industry. The classical inventory literature studies the minimum quantity in the form of lot sizing, economic order quantity, and batch ordering problems (see, e.g., [22]). Recently, Chow et al. [23] investigate how imposition of minimum order quantity affects a fashion supply chain adopting quick response strategy. Their findings suggest that the order constraint has different impacts on different supply chain agents and thus should be set carefully for the sake of the whole supply chain.

\section{Problem Description}

We consider the situation that an online retailer $(R)$ provides a designer platform service (DPS) to an entrant designer $(D)$ to display one of her (throughout the paper, we employ the pronoun "she" for the designer and "he" for the retailer for ease of presentation) own-designed apparel to customers. The retail price of the product, $p>0$, is endogenous and decided by $D$. Customers who are willing to buy the product will preorder at $p$ online before a specified deadline of ordering. The demand of the product, which is reflected by the total quantity of the preorders placed, is price-dependent and is given by

$$
y(p ; x)=z(p) x
$$

where $0 \leq z(p)<+\infty$ and $d z(p) / d p:=z^{\prime}(p)<0$ for all $p \geq 0$, and $x \geq 0$ is a random variable with probability density function $f(x)$ and cumulative distribution function $F(x)$. Without further notification, we assume that $d F(x) / d x=$ $f(x)>0$ for all $x \geq 0$.

The total production cost with production quantity $q$ is given by

$$
C(q)=g+h q,
$$

where $g>0$ is the fixed cost and $h>0$ is the variable cost of production. We note that the unit product cost is given by

$$
c(q)=\frac{C(q)}{q}=\frac{g}{q+h}
$$

and hence

$$
\frac{d c(q)}{d q}=-g q^{-2}<0, \quad \forall q>0
$$

Specifically, $c(q)$ is strictly decreasing in $q$ for all $q>0$. To justify the fixed cost of production, $R$ requires a minimum production quantity (MPQ) $Q>0$, such that if $y(p ; x) \geq Q$, then $R$ will proceed to arrange a manufacturer for production and all the orders placed will be fulfilled, and the customers pay for the products. Otherwise, the product will not be produced and customers will not be charged. Mathematically, the production quantity $q$ is given by

$$
q(p, Q ; x)= \begin{cases}y(p ; x), & \text { if } y(p ; x) \geq Q \\ 0, & \text { o.w. }\end{cases}
$$

We consider that the profit of selling the product is shared by $R$ and $D$ with the former sharing $\alpha$, whilst the latter sharing $(1-\alpha)$ of the total profit, where $0 \leq \alpha \leq 1$. As a remark, different ways of profit allocation under DPS project could result in different optimal solutions of $Q$ and $p$. However, the effects of the allocation schemes are out of the scope of this paper.

For any given $Q>0$ and $p>0$, the profit of $R$ is given by

$$
\begin{aligned}
& \pi(Q, p ; x) \\
& = \begin{cases}\alpha[p y(p ; x)-C(y(p ; x))], & \text { if } y(p ; x) \geq Q \\
0, & \text { o.w, }\end{cases} \\
& = \begin{cases}\alpha[(p-h) z(p) x-g], & \text { if } x z(p) \geq Q \\
0, & \text { o.w. }\end{cases}
\end{aligned}
$$

Taking the expectation of $\pi(Q, p ; x)$ on $x$, the expected profit of $R$ is given by

$$
E[\pi(Q, p)]=\alpha \int_{Q / z(p)}^{\infty}\{(p-h) z(p) x-g\} f(x) d x
$$

The feasibility of DPS depends on whether the total order quantity received is greater than the MPQ. We define the feasibility probability of DPS as the probability of the total order quantity being greater than the MPQ; that is,

$$
\Psi(p, Q)=\operatorname{Pr}\left\{x \geq \frac{Q}{z(p)}\right\}=1-F\left[\frac{Q}{z(p)}\right] .
$$

The sales volume is given by

$$
q(p, Q ; x)= \begin{cases}y(p ; x)=x z(p), & \text { if } x z(p) \geq Q \\ 0, & \text { o.w. }\end{cases}
$$

Correspondingly, the expected sales volume is given by

$$
E[q(p, Q)]=z(p) \int_{\mathrm{Q} / z(p)}^{\infty} x f(x) d x
$$

As a remark, $R$ must be profitable in order for him to participate in DPS. In other words, we need to have $\pi(Q, p ; x)>0$ for all $x \geq Q / z(p)$, which is equivalent to $\alpha[(p-h) z(p) x-g]>0$ for all $x \geq Q / z(p)$. Simplifying 
TABLE 1: The list of scenarios being considered in this paper.

\begin{tabular}{|c|c|c|c|c|}
\hline \multirow{2}{*}{ Scenario } & \multicolumn{2}{|c|}{ Decision sequence } & \multirow{2}{*}{ Follower's problem } & \multirow{2}{*}{ Leader's problem } \\
\hline & Leader & Follower & & \\
\hline $\begin{array}{l}\text { Project-oriented } \\
R \text {-led }\end{array}$ & Retailer & $\begin{array}{c}\text { Project-oriented } \\
\text { designer }\end{array}$ & $\begin{array}{l}\text { For any given } Q \text {, to find } \\
p^{*}=\underset{p \geq 0}{\arg \max }\{\Psi(p, Q)\} \\
\text { s.t. } \alpha \pi(Q, p) \geq 0 \text {, for all } x \geq 0 .\end{array}$ & $\begin{array}{l}\text { Anticipating } p^{*}, \text { to find } \\
Q^{*}=\underset{\mathrm{Q} \geq 0}{\arg \max }\left\{E\left[\pi\left(Q, p^{*}\right)\right]\right\}\end{array}$ \\
\hline $\begin{array}{l}\text { Market-oriented } \\
R \text {-led }\end{array}$ & Retailer & $\begin{array}{c}\text { Market-oriented } \\
\text { designer }\end{array}$ & $\begin{array}{l}\text { For any given } Q \text {, to find } \\
p^{*}=\underset{p \geq 0}{\arg \max }\{E[q(p, Q)]\} \\
\text { s.t. } \alpha \pi(Q, p) \geq 0 \text {, for all } x \geq 0 .\end{array}$ & $\begin{array}{l}\text { Anticipating } p^{*}, \text { to find } \\
Q^{*}=\underset{\mathrm{Q} \geq 0}{\arg \max }\left\{E\left[\pi\left(Q, p^{*}\right)\right]\right\}\end{array}$ \\
\hline $\begin{array}{l}\text { Project-oriented } \\
D \text {-led }\end{array}$ & $\begin{array}{l}\text { Project-oriented } \\
\text { designer }\end{array}$ & Retailer & $\begin{array}{l}\text { For any given } p \text {, to find } \\
Q^{*}=\underset{Q \geq 0}{\arg \max }\{E[\pi(Q, p)]\}\end{array}$ & $\begin{array}{l}\text { Anticipating } Q^{*}, \text { to find } \\
p^{*}=\underset{p \geq 0}{\arg \max }\left\{\Psi\left(p, Q^{*}\right)\right\} \\
\text { s.t. } \alpha \pi\left(Q^{*}, p^{*}\right) \geq 0, \text { for all } x \geq 0\end{array}$ \\
\hline $\begin{array}{l}\text { Market-oriented } \\
D \text {-led }\end{array}$ & $\begin{array}{l}\text { Market-oriented } \\
\text { designer }\end{array}$ & Retailer & $\begin{array}{l}\text { For any given } p \text {, to find } \\
Q^{*}=\underset{\mathrm{Q} \geq 0}{\arg \max }\{E[\pi(Q, p)]\}\end{array}$ & $\begin{array}{l}\text { Anticipating } Q^{*}, \text { to find } \\
p^{*}=\underset{p \geq 0}{\arg \max }\left\{E\left[q\left(p, Q^{*}\right)\right]\right\} \\
\text { s.t. } \alpha \pi\left(Q^{*}, p^{*}\right) \geq 0, \text { for all } x \geq 0\end{array}$ \\
\hline
\end{tabular}

the above yields $(p-h) z(p) x \geq g$ for all $x \geq Q / z(p)$. By putting $Q=x z(p)$, we need to have $(p-h) Q \geq g$ or $p \geq h+g / Q$. Moreover, for any given $Q$ and $p$, if $x<Q / z(p)$ for all $x \geq 0$, then both $D$ and $R$ have zero profit. Therefore, there should exist some $x$ such that $x \geq Q / z(p)$.

For any given pair of $(Q, p)$, the pair of $(Q, p)$ is said to be feasible if (i) $p \geq h+g / Q$ and if (ii) there exists some $x$ such that $x \geq Q / z(p)$. The following summarize condition (i) of $(Q, p)$ being feasible. Let

$$
p_{0}(Q)=h+\frac{g}{Q}
$$

(C1): $p \geq p_{0}(Q)>h$ for any given $Q$.

For condition (ii), the specific condition depends on the exact form of $f(x)$.

\section{The Best Response Decisions of DPS}

There are two decision variables under DPS, namely $Q$ and $p$. The optimal values of $Q$ and $p$ may be different with different decision sequences. Therefore, in this paper we consider two scenarios, one with $R$ making the decision first, whilst the other with $D$ being the first mover. A game with $R$ as the first mover is referred to as an $R$-led (game), whereas the one with $D$ as the first mover is referred to as a $D$-led (game). The objectives of the two parties are also different. Ris to maximize his expected profit, which must be nonnegative. For $D$, whether her product can be sold or not, or how many customers will buy her product are more important than the profit she is to earn from selling her product. Therefore, we consider the following two types of $D$ whose objectives are (1) maximizing the feasibility probability of DPS project; and (2) maximizing the expected market size, which is equivalent to maximizing the expected sales volume. We refer to $D$, having the first objective, as "Project-oriented Designer," whilst the one with the second objective is "Market-oriented Designer." Table 1 summarizes the scenarios that are considered in this paper.
As $D$ and $R$ make decisions sequentially, we apply the Stackelberg game in analyzing the model in which the first mover acts as the game leader and the late mover acts as the game follower. Moreover, we apply backward induction to obtain the optimal solutions of the players in the game. Propositions 1 to 4 provide the best response solutions to various scenarios.

Proposition 1. Under the "Project-oriented R-led" scenario:

(a) for any fixed $Q>0$, the DPS feasibility probability, $\Psi(Q, p)$, is decreasing in $p$ for $p>0$, and the best response price that maximizes $\Psi(Q, p)$ is uniquely given by $p_{1}^{*}(Q)=p_{0}(Q)$,

(b) if the best response MPQ that maximizes the expected profit of $R$ (denoted by $Q_{1}{ }^{*}$ ) exists and satisfies the firstorder optimality condition, it is given by

$$
Q_{1}{ }^{*}=\arg \left\{Q \geq 0: Q z\left(h+\frac{g}{Q}\right)+g z^{\prime}\left(h+\frac{g}{Q}\right)=0\right\} \text {. }
$$

Proof. All proofs are relegated in the Appendix.

Proposition 2. Under the "Market-oriented R-led" scenario:

(a) for any fixed $Q>0$, the expected sales volume, $E[q(Q, p)]$, is decreasing in $p$ for $p>0$, and the best response price that maximizes the expected market size is uniquely given by

$$
p_{2}^{*}(Q)=p_{0}(Q)
$$

(b) if the best response MPQ that maximizes the expected profit of $R$ (denoted by $Q_{2}{ }^{*}$ ) exists and satisfies the firstorder optimality condition, it is given by

$$
Q_{2}{ }^{*}=\arg \left\{Q>0: Q z\left(h+\frac{g}{Q}\right)+g z^{\prime}\left(h+\frac{g}{Q}\right)=0\right\} \text {. }
$$


Propositions 1 and 2 assert that when $R$ is the first mover in DPS, the best response decisions are the same whether $D$ is project-oriented or market-oriented. In particular, under the two $R$-led scenarios, it is always optimal for $D$ to set the retail price as low as possible [i.e., $\left.p_{0}(Q)\right]$. Such result is natural as the objective of $D$ is not related to the expected profit she is to earn from DPS.

Proposition 3. Under the "Project-oriented D-led" scenario:

(a) for any fixed $p>p_{0}$

(a-i) $E[\pi(Q, p)]$ is strictly concave in $Q$ if and only if

$$
(p-h) z(p) f\left[\frac{Q}{z(p)}\right]+[(p-h) Q-g] f^{\prime}\left[\frac{Q}{z(p)}\right]>0,
$$

(a-ii) a sufficient condition for strict concavity of $E[\pi(Q, p)]$ is $f^{\prime}[Q / z(p)] \geq 0$ for all $Q>0$; and

(a-iii) if $f^{\prime}[Q / z(p)] \geq 0$, the best response MPQ that maximizes the expected profit of $R$ is uniquely given by $Q_{3}{ }^{*}(p)=g /(p-h)$;

(b) if the best response price that maximizes the feasibility probability of DPS exists and satisfies the first-order optimality condition, it is given by

$$
p_{3}{ }^{*}=\arg \left\{p>p_{0}: z(p)+(p-h) z^{\prime}(p)=0\right\} \text {. }
$$

Proposition 4. Under the "Market-oriented D-led" scenario:

(a) for any fixed $p>p_{0}$,

(a-i) $E[\pi(Q, p)]$ is strictly concave in $Q$ if and only if

$$
(p-h) z(p) f\left[\frac{Q}{z(p)}\right]+[(p-h) Q-g] f^{\prime}\left[\frac{Q}{z(p)}\right]>0,
$$

(a-ii) a sufficient condition for strict concavity of $E[\pi(Q, p)]$ is $f^{\prime}[Q / z(p)] \geq 0$ for all $Q>0$; and

(a-iii) if $f^{\prime}[Q / z(p)] \geq 0$, the best response MPQ that maximizes the expected profit of $R$ is uniquely given by

$$
Q_{4}^{*}(p)=\frac{g}{p-h}
$$

(b) if the best response price, that maximizes the expected sales volume, exists and satisfies the first-order optimality condition, it is given by

$$
p_{4}^{*}=\arg \left\{p>p_{0}: \zeta(p)=0\right\}
$$

where

$$
\begin{aligned}
\zeta(p)=(p-h) z^{\prime}(p) & \left((p-h)^{2} z^{2}(p) \int_{g /[(p-h) z(p)]}^{\infty} x f(x) d x\right. \\
& \left.+g^{2} f\left[\frac{g}{(p-h) z(p)}\right]\right) \\
& +g^{2} z(p) f\left(\frac{g}{(p-h) z(p)}\right) .
\end{aligned}
$$

On the other hand, Propositions 3 and 4 indicate that when $D$ is the first mover in DPS (i.e., the two $D$-led scenarios), if the best response MPQ exists and is positive, then we have $Q_{3}{ }^{*}(p)=Q_{4}{ }^{*}(p)=g /(p-h)$.

As a remark, it is the lowest MPQ that promises nonnegative profit. In addition, the smaller the MPQ, the higher the unit price that $D$ should meet, which means more profit from excess order to MPQ. Therefore, regardless of the objective of $D$, the best response function of $R$ is given by

$$
Q_{0}(p)=\frac{g}{p-h}
$$

Notice that $g$ is the fixed cost for production, whilst $(p-h)$ can be viewed as the unit "profit margin" for each piece of apparel under DPS. In other words, the retailer should set the MPQ in the way that such a corresponding profit can justify the fixed production cost.

Moreover, we note that the existence of the best response retail price and the best response MPQ depend on the functions $z(p)$ and $f(x)$; there may be situations where no optimal solutions exist. There is no general condition to ensure the existence of them. Propositions 1 to 4 show the analytical forms of the optimal retail price and the optimal MPQ in case they exist.

\section{Analysis with Specific Demand Functions}

To obtain more insight, we consider specific forms of $z(p)$ and $F(x)$ in this section. Specifically, we consider that $x$ follows a uniform distribution $U[l, u]$, where $0<l<u$. For $z(p)$, we consider two specific types that are commonly adopted in the literature (e.g., [17]), namely

(1) additive form:

$$
z_{A}(p)= \begin{cases}a-b p, & \text { for } 0<p<\frac{a}{b} \\ 0, & \text { for } p \geq \frac{a}{b}\end{cases}
$$

for $a>b h>0$ (as $p>h$, if $a>b h$ does not hold, then $p>a / b$ and $\left.z_{A}(p)=0\right)$; and

(2) multiplicative form: $z_{M}(p)=a p^{-b}$ for $a>0$ and $b>$ 0 . 
TABLE 2: Equilibrium decisions with additive form demand function $z_{A}(p)=a-b p$.

\begin{tabular}{lccc}
\hline Scenario & Condition(s) for existence of the equilibrium & Equilibrium $Q$ & \\
\hline $\begin{array}{l}\text { Project-oriented } R \text {-led } \\
\text { Market-oriented } R \text {-led }\end{array}$ & $g<(a-b h)^{2} u / 4 b$ & $2 b g /(a-b h)$ & Equilibrium $p$ \\
Project-oriented $D$-led & $g<(a-b h)^{2} u / 4 b$ and $p_{L, 4 A}<\hat{p}_{4 A}<p_{H, 4 A}$ & $g /\left(\hat{p}_{4 A}-h\right)$ & Given by $\widehat{p}_{4 A}$ \\
Market-oriented $D$-led & $g h) / 2 b$ \\
\hline
\end{tabular}

Next, we study the equilibriums with two demand forms separately.

5.1. Additive Form Demand Function. With $z(p)=z_{A}(p)=$ $a-b p$, Propositions 5 to 7 detail the equilibrium decisions and the conditions for the existence of the equilibrium decisions for DPS under different scenarios.

Proposition 5. For the two "R-led" scenarios:

(a) there exists feasible $(Q, p)$ only if $g<(a-b h)^{2} u / 4 b$ and $Q>g b /(a-b h)$,

(b) for $g<(a-b h)^{2} u / 4 b$, the expected profit of $R$ is unimodal in $Q$ for all $Q>0$, and the equilibrium $M P Q$ is uniquely given by $Q_{1 A}{ }^{*}=2 g b /(a-b h)>$ $g b /(a-b h)$. Correspondingly, the equilibrium price is uniquely given by $p_{1 A}{ }^{*}=(a+b h) / 2 b>h$.

Let $\Delta=u\left[u(a-b h)^{2}-4 b g\right], p_{L, 3 A}=(a+b h) / 2 b-\sqrt{\Delta} / 2 u b$, and $p_{H, 3 A}=(a+b h) / 2 b+\sqrt{\Delta} / 2 u b$.

Proposition 6. For the "Project-oriented D-led" scenario:

(a) the best response MPQ of $R$ is given by $Q_{0}(p)=g /(p-$ $h)$ and the pair $\left(Q_{0}(p), p\right)$ is feasible only if $g<u(a-$ $b h)^{2} / 4 b$,

(b) for $g<u(a-b h)^{2} / 4 b$, the DPS feasibility probability is strictly concave in $p$, the equilibrium retail price is $p_{3 A}{ }^{*}=(a+b h) / 2 b>h$, and the equilibrium MPS is $Q_{3 A}{ }^{*}=2 b g /(a-b h)$.

Proposition 7. For the "Market-oriented D-led" scenario, for $g<u(a-b h)^{2} / 4 b$ :

(a) the expected sales volume function $E\left[q\left(p, Q_{0}(p)\right)\right]$ given by (10) is strictly concave in $p$,

(b) let $\widehat{p}_{4 A}=\arg _{p}\left\{g^{2}(2 a+b h-3 b p)-b u^{2}(p-h)^{3}(a-b p)^{2}=\right.$ $0\}$. If $p_{L, 3 A}<\widehat{p}_{4 A}<p_{H, 3 A}$, then the equilibrium $p$ is $\widehat{p}_{4 A}$ and the equilibrium MPS is $Q_{4 A}{ }^{*}=g /\left(\widehat{p}_{4 A}-h\right)$; otherwise, $\left(Q_{0}(p), p\right)$ is not feasible for all $p>0$.

Table 2 summarizes the equilibrium decisions under various scenarios and the respective conditions required.

With $z(p)=z_{A}(p)=a-b p$, we found that $g-(a-$ $b h)^{2} u / 4 b<0$ is the common condition for the existence of the equilibrium solutions for all scenarios. As $g-(a-$ $b h)^{2} u / 4 b<0$ is strictly increasing with $g, h$, and $b$ and is strictly decreasing with $a$ and $u$, the condition $g-(a-$ $b h)^{2} u / 4 b<0$ implies that the equilibrium decision may not exist when $g, h$, and/or $b$ are big, and/or $a$ and/or $u$ are small. We argue such results as follows. When $g$ and/or $h$ are big, it is not profitable for $R$ under DPS, and hence, $R$ will not participate with DPS. When $b$ is big, the product demand is very sensitive to the price such that it is not possible to set a price to make $q$ no less than MPQ and $R$ with profitable. When $a$ and/or $u$ are small, the demand base is small. Therefore, again, it is not possible to set a price to make $q$ no less than MPQ and $R$ profitable.

For the equilibrium decisions, they are the same for the scenarios Project-oriented $R$-led, Market-oriented $R$-led, and Project-oriented $D$-led. However, the equilibrium decisions of the Market-oriented $D$-led scenario are different from the other three scenarios. Moreover, for the Market-oriented $D$ led scenario, there is an extra condition, $p_{L, 4 A}<\widehat{p}_{4 A}<$ $p_{H, 4 A}$ for the existence of the equilibrium solutions. This condition gives an upper bound and a lower bound of the equilibrium $p$. We argue such results as follows. Being an entrant designer, $D$ cares less about maximizing her own profit. Rather, she aims at either maximizing the expected sales volume, or the feasibility probability of the DPS. Both objectives can be fulfilled by maximizing the demand, which is also favorable to the profit-maximizing $R$. In other words, the objective of $D$ is consistent with that of $R$; therefore the effect of decision sequence becomes negligible. Besides, whether $D$ is project- or market-oriented, it is also optimal for her to set $p$ as small as possible to maximize the market size whilst keeping $R$ profitable. From the perspective of $R$, as discussed in the previous section, the optimal MPQ is the one that barely justifies the fixed production cost. This reflects that it is always optimal for him to make DPS feasible so that he can have the opportunity to gain profit. Hence, the equilibrium decisions are almost the same under different decision sequences and different objectives of $D$ when there is no limitation on setting $p$. However, because of the extra condition for the equilibrium $p$, the equilibrium decisions for the Market-oriented $D$-led scenario are different from the other three scenarios.

5.2. Multiplicative Form Demand Function. With $z(p)=$ $z_{M}(p)=a p^{-b}$, Propositions 8 to 10 detail the equilibrium decisions and the conditions for the existence of the equilibrium decisions for DPS under different scenarios.

Proposition 8. For the two "R-led" scenarios

(a) the best response retail price of $D$ is given by $p_{0}(Q)=$ $h+g / Q$;

(b) for $0<b \leq 1$, the expected profit of $R, E\left[\pi_{1 M}(Q)\right]$, is strictly decreasing in $Q$ and $E\left[\pi_{1 M}(0)\right]=+\infty$; and 
TABLE 3: Equilibrium decisions with multiplicative form demand function $z_{M}(p)=a p^{-b}$.

\begin{tabular}{lccc}
\hline Scenario & Condition $(s)$ for existence of equilibrium & Equilibrium $Q$ & \\
\hline $\begin{array}{l}\text { Project-oriented } R \text {-led } \\
\text { Market-oriented } R \text {-led }\end{array}$ & $b>1$ and $g(b-1)[h b /(b-1)]^{b} / a h<u$ & $(b-1) g / h$ & Equilibrium $p$ \\
$\begin{array}{l}\text { Project-oriented } D \text {-led } \\
\text { Market-oriented } D \text {-led }\end{array}$ & $b>1$ and $l<g(b-1)[h b /(b-1)]^{b} / a h<u$ & & \\
\hline
\end{tabular}

(c) for $b>1$, the expected profit of the retailer is unimodal in $Q$ for all $Q>0$;

(c-i) if $g(b-1)[h b /(b-1)]^{b} / a h \geq u,\left(Q, p_{0}(Q)\right)$ is infeasible for all $Q>0$; and

(c-ii) otherwise, the equilibrium MPQ and the equilibrium $p$ are given by $Q_{1 M}{ }^{*}=(b-1) g / h$ and $p_{1 M}{ }^{*}=b h /(b-1)$, respectively.

Let $\Delta_{3 A}=u\left[u(a-b h)^{2}-4 b g\right], p_{L, 3 A}=(a+b h) / 2 b-$ $\sqrt{\Delta_{3 A}} / 2 u b$, and $p_{H, 3 A}=(a+b h) / 2 b+\sqrt{\Delta_{3 A}} / 2 u b$. Moreover, let

$$
K_{3 M}(p)=\frac{g p^{b}}{a(p-h)}<u
$$

and denote $p_{3 M l}{ }^{\prime}, p_{3 M u}{ }^{\prime}, p_{3 M u}{ }^{\prime \prime}, p_{3 M u}{ }^{\prime \prime \prime}, p_{3 M l}{ }^{\prime \prime}, p_{3 M l}{ }^{\prime \prime \prime}$, $p_{3 M l}{ }^{\prime}$, and $p_{3 M u}$ that satisfy (i) $p_{3 M l}>p_{3 M u}>h$, (ii) $K_{3 M}\left(p_{3 M l}{ }^{\prime}\right)=l$ and (iii) $K_{3 M}\left(p_{3 M u}{ }^{\prime}\right)=u$; and $p_{3 M u}{ }^{\prime \prime}{ }^{\prime \prime}$, $p_{3 M u}{ }^{\prime \prime \prime}, p_{3 M l}{ }^{\prime \prime}$, and $p_{3 M l}{ }^{\prime \prime \prime}$ satisfy (i) $h<p_{3 M u} "<p_{3 M l} " \leq$ $p_{3 M l}{ }^{\prime \prime \prime}<p_{3 M u}{ }^{\prime \prime \prime}$, (ii) $K_{3 M}\left(p_{3 M u}{ }^{\prime \prime}\right)=K_{3 M}\left(p_{3 M u}{ }^{\prime \prime \prime}\right)=u$, (iii) $K_{3 M}\left(p_{3 M l}{ }^{\prime \prime}\right)=K_{3 M}\left(p_{3 M l}{ }^{\prime \prime \prime}\right)=l$, (iv) $l<K_{3 M}(p)<u$ for all $p_{3 M u}{ }^{\prime \prime}<p<p_{3 M l}$ " or $p_{3 M l}{ }^{\prime \prime \prime}<p<p_{3 M u}{ }^{\prime \prime \prime}$, and (v) $K_{3 M}(p) \leq l$ for all $p_{3 M l}{ }^{\prime \prime}<p<p_{3 M l}{ }^{\prime \prime \prime}$.

Proposition 9. For the "Project-oriented D-led" scenario:

(a) the best response MPQ of $R$ is $Q_{0}(p)=g /(p-h)$;

(b) for $b \leq 1$, the feasibility probability $\Psi(p)$ is strictly increasing in $p$ for all $p_{3 M u}{ }^{\prime}<p<p_{3 M l}{ }^{\prime}$ and $\Psi(l)=$ 1 for all $p \geq p_{3 M l}{ }^{\prime}$,

(c) for $b>1$,

(c-i) if $(g(b-1) / a h)(h b /(b-1))^{b} \geq u$, then the pair $\left(Q_{3 M}^{*}(p), p\right)$ is infeasible;

(c-ii) if $l<(g(b-1) / a h)(h b /(b-1))^{b}<u$, then $\Psi(p)$ is unimodal in $p$ and is maximized at $p=h b /(b-$ $1)$;

(c-iii) if $(g(b-1) / a h)(h b /(b-1))^{b}<l$, then $\Psi(p)$ is strictly increasing in $p$ for all $p_{3 M u}{ }^{\prime \prime}<p<$ $p_{3 M l}{ }^{\prime \prime}$, is strictly decreasing in $p$ for all $p_{3 M l}{ }^{\prime \prime \prime}<$ $p<p_{3 M u}{ }^{\prime \prime \prime}$, and $\Psi_{3 M}(p)=1$ for all $p_{3 M l}{ }^{\prime \prime}<p<$ $p_{3 M l}$.

Proposition 9 asserts that for the Project-oriented $D$-led scenario, the equilibrium decision exists only if $b>1$ and $l<(g(b-1) / a h)(h b /(b-1))^{b}<u$, and the equilibrium $Q$ and equilibrium $p$ are given by $(b-1) g / h$ and $b h /(b-1)$, respectively.
Proposition 10. Under the "Market-oriented D-led" scenario:

(a) for $b \leq 1$, the expected sales volume $E\left[q\left(p, Q_{0}(p)\right)\right]$ is strictly increasing in $p$ for all $p_{3 M u}{ }^{\prime}<p<p_{3 M l}{ }^{\prime}$, and $E\left[q\left(p, Q_{0}(p)\right)\right]=(u-l) / 2$ for all $p \geq p_{3 M l}$.

(b) for $b>1$,

(b-i) if $l<(g(b-1) / a h)(h b /(b-1))^{b}<u$, then $E\left[q\left(p, Q_{0}(p)\right)\right]$ is unimodal in $p$ and is maximized at $p=h b /(b-1)$; and

(b-ii) if $(g(b-1) / a h)(h b /(b-1))^{b}<l$, then $E\left[q\left(p, Q_{0}(p)\right)\right]$ is strictly increasing in $p$ for all $p_{3 M u}{ }^{\prime \prime}<p_{u I}<p_{3 M l}{ }^{\prime \prime}$, is strictly decreasing in $p$ for all $p_{3 M l}{ }^{\prime \prime \prime}<p<p_{3 M u}{ }^{\prime \prime \prime}$, and $\Psi_{3 M}(p)=1$ for all $p_{3 M l} \|<<p_{3 M l}$.'.

Proposition 10 asserts that for the Market-oriented $D$-led scenario, the equilibrium decision exists only if $b>1$ and $l<(g(b-1) / a h)(h b /(b-1))^{b}<u$, and the equilibrium $Q$ and equilibrium $p$ are given by $(b-1) g / h$ and $b h /(b-1)$, respectively.

Table 3 summarizes the best response solutions under various scenarios and the respective conditions required.

With $z(p)=z_{M}(p)=a p^{-b}$, we found that the optimal solutions are almost the same whether $D$ is project- or market-oriented. They are also independent of the decision sequence. Similar to the case with $z(p)=z_{A}(p)=a-b p$, we argue such results as follows. Being an entrant designer, $D$ cares less about maximizing her own profit. Rather, she aims at either maximizing the expected sales volume or the feasibility probability of the DPS. Both objectives can be fulfilled by maximizing the demand, which is also favorable to the profit-maximizing $R$. In other words, the objective of $D$ is consistent with that of $R$; therefore, the effect of decision sequence becomes negligible. Besides, whether $D$ is projector market-oriented, it is also optimal for her to set $p$ as small as possible to maximize the market size whilst keeping $R$ profitable. Hence, the optimal solutions are almost the same under different decision sequences and different objectives of $D$. From the perspective of $R$, as discussed in the previous section, the optimal MPQ is the one that barely justifies the fixed production cost. This reflects that it is always optimal for him to make DPS feasible so that he can have the opportunity to gain profit.

The optimality conditions provide some other insights to the equilibrium decisions. To be specific, $b>1$ is required in order to have equilibrium decisions for all four scenarios: $g(b-1)[h b /(b-1)]^{b} / a h<u$ is required for the two $R$-led scenarios and $l<g(b-1)[h b /(b-1)]^{b} / a h<u$ is required for 
the two $D$-led scenarios. As $l$ does not include the equilibrium $Q$ and the equilibrium $p$, the value of $l$ is irrelevant in deriving the equilibrium decisions for the two $R$-led scenarios, but $l$ becomes relevant in deriving the equilibrium decisions for the two $D$-led scenarios. Moreover, it is more probable an equilibrium decision could be derived for two $R$-led scenarios than for the two $D$-led scenarios.

\section{Discussion}

This paper concerns a service platform provided by an online retailer to an entrant designer. By formulating the problem as various Stackelberg games, we first explore the best response retail price of the entrant designer and the best response MPQ of the retailer, under different designer's objectives and decision sequences in a general demand function setting, and then we explore the equilibrium decisions of the games, under different designer's objectives and decision sequences by considering two specific demand function setting structures. Essentially, the retailer should set the MPQ that justifies the fixed production cost, whilst the entrant designer should set the retail price as low as possible to enlarge the market demand whilst the retail is still profitable.

The current paper bares several limitations including but not limited to the below. First, this paper considers the simple case that the product is sold at a fixed price at all quantities, whereas there can be various ways in organizing the group selling; a possible extension is to explore how different pricing schemes may bring benefits to all parties in the system. For instance, a pricing scheme with quantity discount, that is, different levels of discounts at different total order quantities is a good candidate for future research. Second, this paper considers the system with single designer and single platform. The literature in crowdsourcing suggests that competition among source providers, which is commonly observed in reality, is good for resource allocation. Accordingly, this study can be extended to explore the effect of competition amongst multiple designers and/or platforms. For other extensions, it is interesting to explore the optimal solutions of the DPS when a well-established designer who cares about profit maximization is involved.

\section{Appendix}

\section{Mathematical Proofs}

Note: for brevity, we omit one of the variables $p$ or $q$ in $E[q(p, Q)], E[\pi(Q, p)]$, and $\Psi(p, Q)$ in the proofs.

Proof of Proposition 1. Under the "Project-oriented R-led" scenario:

(a) for any given $Q>0$, the DPS feasibility probability is given by $\Psi(p)=1-F[Q / z(p)]$, and we have

$$
\begin{gathered}
\frac{d \Psi(p)}{d p}=f\left[\frac{Q}{z(p)}\right]\left[\frac{Q z^{\prime}(p)}{z^{2}(p)}\right] \leq 0, \quad \text { as } z^{\prime}(p)<0 \\
f(x) \geq 0, \quad \forall x \geq 0 .
\end{gathered}
$$

Thus, the optimal retail price that maximizes the expected sales volume is the smallest possible value of the retail price, which is equal to $p_{0}(Q)$ by $(C 1)$.

(b) anticipating that $D$ would set the retail price as $p_{2}{ }^{*}(Q)=p_{0}(Q)$, the retailer's expected profit when having $Q$ as the MPQ and the corresponding first derivative in $Q$ are given by the below, respectively:

$$
\begin{gathered}
E[\pi(Q)]=\alpha \int_{Q / z\left[p_{2}{ }^{*}(Q)\right]}^{\infty}\left[\left(\frac{g}{Q}\right) z\left(p_{2}{ }^{*}(Q)\right) x-g\right] f(x) d x \\
=\alpha g\left[\frac{z(h+g / Q)}{Q} \int_{Q / z(h+g / Q)}^{\infty} x f(x) d x\right. \\
\left.+F\left(\frac{Q}{z(h+g / Q)}\right)-1\right],
\end{gathered}
$$

$$
\begin{aligned}
d E \frac{[\pi(Q)]}{d Q}= & -\left(\frac{\alpha g}{Q^{3}}\right)\left[Q z\left(h+\frac{g}{Q}\right)+g z^{\prime}\left(h+\frac{g}{Q}\right)\right] \\
& \times \int_{Q / z(h+g / Q)}^{\infty} x f(x) d x .
\end{aligned}
$$

By the participation constraint of $R$, that is, $\pi(Q, p ; x)>0$, we have $E[\pi(Q)]>0$. Therefore, $\int_{Q / z(h+g / Q)}^{\infty} x f(x) d x>(Q / z(h+$ $g / Q))(1-F(Q / z(h+g / Q)))>0$. From (A.3), we then have $d E[\pi(Q)] /\left.d Q\right|_{\mathrm{Q}=\mathrm{Q}_{2}}{ }^{*}=0 \Leftrightarrow \mathrm{Q}_{2}{ }^{*} z\left(h+g / \mathrm{Q}_{2}{ }^{*}\right)+g z^{\prime}(h+$ $\left.g / Q_{2}{ }^{*}\right)=0$.

Proof of Proposition 2. Under the "Market-oriented R-led" scenario:

(a) for any given $Q>0$, the expected sales volume is given by $E[q(p)]=z(p) \int_{\mathrm{Q} / z(p)}^{\infty} x f(x) d x$ and we have $d E[q(p)] / d p=z^{\prime}(p)\left[\int_{\mathrm{Q} / z(p)}^{\infty} x f(x) d x+\right.$ $\left.Q^{2} f[Q / z(p)] / z^{2}(p)\right] \leq 0$, as $z^{\prime}(p)<0$, and $f(x) \geq$ 0 for all $x \geq 0$. Thus, the optimal retail price that maximizes the expected sales volume is the smallest possible value of the retail price, which is equal to $p_{0}(Q)$ by $(\mathrm{C} 1)$.

(b) same as Proposition 1(b),

Proof of Proposition 3. (a) For any fixed $p>p_{0}$, the expected profit of $R$ is given by

$$
\begin{aligned}
E[\pi(Q)]=\alpha \int_{Q / z(p)}^{\infty}[(p-h) z(p) x-g] f(x) d x \\
=\alpha\left((p-h) z(p) \int_{Q / z(p)}^{\infty} x f(x) d x\right. \\
\left.+g F\left[\frac{Q}{z(p)}\right]+g\right) .
\end{aligned}
$$


The first and second derivatives of $E[\pi(Q)]$ w.r.t. $Q$ can be easily derived as follows, respectively:

$$
\begin{aligned}
& \frac{d E[\pi(Q)]}{d Q}=-\frac{\alpha f[Q / z(p)][(p-h) Q-g]}{z(p)}, \\
& \frac{d^{2} E[\pi(Q)]}{d Q^{2}} \\
& \quad=-\alpha\left((p-h) z(p) f\left[\frac{Q}{z(p)}\right]\right. \\
& \left.\quad+[(p-h) Q-g] f^{\prime}\left[\frac{Q}{z(p)}\right]\right) \times\left(z^{2}(p)\right)^{-1} .
\end{aligned}
$$

(a-i) A direct observation from (A.6). (a-ii) If $f^{\prime}[Q / z(p)] \geq 0$ for all $Q>0$, then

$$
\begin{aligned}
& (p-h) z(p) f\left[\frac{Q}{z(p)}\right]+[(p-h) Q-g] f^{\prime}\left[\frac{Q}{z(p)}\right] \\
& \quad \geq(p-h) z(p) f\left[\frac{Q}{z(p)}\right]>0 .
\end{aligned}
$$

Noticed that $p \geq p_{0}(Q)>h$ and $f(x)>0$. So by (ai), $E[\pi(Q)]$ is strictly concave in $Q$. (a-iii) A direct result by solving the first-order condition $d E[\pi(Q)] / d Q=0$ from (A.5).

(b) Anticipating that the retailer sets the minimum processing quantity as $Q_{3}{ }^{*}(p)$, the probability of DPS feasibility is given by

$$
\Psi(p)=1-F\left[\frac{Q_{3}{ }^{*}(p)}{z(p)}\right]=1-F\left[\frac{g}{(p-h) z(p)}\right] .
$$

The first derivative of $\Psi(p)$ is

$$
\begin{aligned}
\frac{d \Psi(p)}{d p}= & {\left[\frac{g}{(p-h)^{2} z^{2}(p)}\right]\left[z(p)+(p-h) z^{\prime}(p)\right] } \\
& \times f\left[\frac{g}{(p-h) z(p)}\right] .
\end{aligned}
$$

The first-order condition $d \Psi\left(p_{3}{ }^{*}\right) / d p=0$ is equivalent to $z\left(p_{3}{ }^{*}\right)+\left(p_{3}{ }^{*}-h\right) z^{\prime}\left(p_{3}{ }^{*}\right)=0$ by direct observation from (A.9).

Proof of Proposition 4. (a) Same as the proofs for Proposition 3(a).

(b) Anticipating that the retailer sets the minimum processing quantity as $Q_{4}{ }^{*}(p)$, the expected sales volume is given by $E[q(p)]=z(p) \int_{g /[(p-h) z(p)]}^{\infty} x f(x) d x$.

It can be easily shown that the first derivative of $E[q(p)]$ is in the form:

$$
\frac{d E[q(p)]}{d p}=\frac{\zeta(p)}{\left[(p-h)^{3} z^{2}(p)\right]}
$$

The optimal price that maximizes the expected sales volume (denoted by $p_{4}{ }^{*}$ ), if exists, should satisfy the first-order condition: $d E\left[q\left(p_{4}{ }^{*}\right)\right] / d p=0$, which is equivalent to $\zeta(p)=$ 0 by direct observation from (A.10).

Proof of Proposition 5. (a) From (C1), we need to have $p \geq h+$ $g / Q$. By the property of $z_{A}(p)$, we also require $0<p<a / b$. Considering both together we have $Q>g b /(a-b h)$. Next, there exists some $x$ such that $x \geq K_{1 A}(Q)$, where $K_{1 A}(Q)=$ $\mathrm{Q} / z_{A}\left(p_{1 A}{ }^{2}\right)$. Since $l<x<u$, we need to have $K_{1 A}(Q)<u$, which is equivalent to $Q^{2}-(a-b h) u Q+b g u<0$. We prove by contradiction that $g \leq(a-b h)^{2} u / 4 b$. Suppose $g>(a-$ $b h)^{2} u / 4 b$. Then

$$
\begin{aligned}
Q^{2}-( & (a-b h) u Q+b g u \\
> & Q^{2}-(a-b h) u Q+\frac{(a-b h)^{2} u^{2}}{4} \\
& =\left[Q-\frac{(a-b h) u}{2}\right]^{2} \geq 0 .
\end{aligned}
$$

A contradiction! So we need to have $g \leq(a-b h)^{2} u / 4 b$.

(b) The profit of the retailer when setting MPQ as $Q$ is given by

$$
\begin{aligned}
& \pi_{1 A}(Q) \\
& = \begin{cases}{\left[p_{1 A}{ }^{*}(Q)-h\right] x z_{A}\left[p_{1 A}{ }^{*}(Q)\right]-g,} & \text { for } x \geq K_{1 A}(Q), \\
0, & \text { o.w., }\end{cases}
\end{aligned}
$$

where $p_{1 A}{ }^{*}(Q)=p_{0}(Q)=h+g / Q$ by Propositions 1 and 2 . Taking expectation, we have

$$
\begin{aligned}
& E\left[\pi_{1 A}(Q)\right] \\
& =\int_{K_{1 A}(Q)}^{u}\left(\left[p_{1 A}{ }^{*}(Q)-h\right] x z_{A}\left[p_{1 A}{ }^{*}(Q)\right]-g\right) f(x) d x \\
& =\frac{g\left[u-K_{1 A}(Q)\right]}{(u-l)}\left(\left[\frac{a-b h}{Q}-\frac{b g}{Q^{2}}\right]\left[\frac{u+K_{1 A}(Q)}{2}\right]-1\right) .
\end{aligned}
$$

The first and second derivative of $E\left[\pi_{1 A}(Q)\right]$ can be easily derived as follows, respectively:

$$
\begin{aligned}
& \frac{d E\left[\pi_{1 A}(Q)\right]}{d Q}=\frac{g\left[u^{2}-K_{1 A}^{2}(Q)\right][-(a-b h) Q+2 b g]}{2(u-l) Q^{3}} \\
& \frac{d^{2} E\left[\pi_{1 A}(Q)\right]}{d Q^{2}}=\frac{g\left[u^{2}-K_{1 A}^{2}(Q)\right][(a-b h) Q-3 b g]}{(u-l) Q^{4}} .
\end{aligned}
$$

From (A.15), for $Q>0, d^{2} E\left[\pi_{1 A}(Q)\right] / d Q^{2}<0 \Leftrightarrow Q<$ $3 b g /(a-b h)\left(\right.$ as $\left.u^{2}>K_{1 A}{ }^{2}(Q)\right)$. Thus, $E\left[\pi_{1 A}(Q)\right]$ is strictly concave for $Q$ in $0<Q<3 b g /(a-b h)$ and is convex for 
$Q \geq 3 b g /(a-b h)$. From (A.14), $d E\left[\pi_{1 A}(Q)\right] / d Q=0 \Leftrightarrow$ $3 b g /(a-b h)>Q=2 b g /(a-b h)>0$ (i.e., a unique $Q>0$ satisfying $\left.d E\left[\pi_{1 A}(Q)\right] / d Q=0\right)$. Therefore, $E\left[\pi_{1 A}(Q)\right]$ is unimodal in $Q$ for $Q>0$. Thus, $Q_{1 A}{ }^{*}=2 b g /(a-b h)$ is unique. Then, by direct manipulation, $p_{1 A}{ }^{*}=p_{1 A}{ }^{*}\left(Q_{1 A}{ }^{*}\right)=$ $(a+b h) / 2 b$.

Proof of Proposition 6. Under the "Project-oriented D-led" scenario with $z(p)=z_{A}(p)=a-b p$ :

(a) by Proposition 3, the optimal MPQ is $Q_{0}(p)=g /(p-$ $h)$, which satisfies (C1). Since $l \leq x \leq u$ and to ensure $\left(Q_{0}(p), p\right)$ being feasible, we need to have $Q_{0}(p) / z_{A}(p)<u$, which is equivalent to

$$
\eta(p)=u b p^{2}-u(a+b h) p+u h a+g<0 .
$$

(b) Suppose $g<u(a-b h)^{2} / 4 b$. For any given $p_{L, 3 A}<p<$ $p_{H, 3 A}$, the DPS feasibility probability is given by

$$
\begin{aligned}
\Psi_{3}(p) & =1-F\left[\frac{Q_{3 A}{ }^{*}(p)}{z_{A}(p)}\right] \\
& =-\frac{g}{(u-l)(p-h)(a-b p)}+\left[1+\frac{u}{(u-l)}\right] .
\end{aligned}
$$

The first and second derivatives of $\Psi_{3}(p)$ w.r.t. $p$ can be derived easily as follows:

$$
\begin{aligned}
\frac{d \Psi(p)}{d p}= & \frac{g}{(u-l)(p-h)^{2}(a-b p)^{2}}(a+h b-2 b p), \\
\frac{d^{2} \Psi(p)}{d p^{2}}= & -\frac{2 g}{(u-l)(p-h)^{3}(a-b p)^{3}} \\
& \times\left[b(p-h)(a-b p)+(a+h b-2 b p)^{2}\right]<0,
\end{aligned}
$$

for all $p_{L, 3 A}<p<p_{H, 3 A}$. So $\Psi_{3}(p)$ is strictly concave in $p$ for $p_{L, 3 A}<p<p_{H, 3 A}$. Solving the first-order condition, from (A.18), we have the optimal retail price: $p_{3 A}{ }^{*}=(a+h b) / 2 b$, which satisfies the constraint as $p_{L, 3 A}<p_{3 A}{ }^{*}<p_{H, 3 A}$. Correspondingly, the optimal MPS in this case is: $Q_{3 A}{ }^{*}=$ $Q_{0}\left(p_{3 A}{ }^{*}\right)=2 b g /(a-b h)$

Proof of Proposition 7. Under the "Market-oriented D-led" scenario with $z(p)=z_{A}(p)=a-b p$ :

(a) For $g<u(a-b h)^{2} / 4 b$, for any given $p_{L, 3 A}<p<$ $p_{H, 3 A}$, the expected sales volume is given by

$$
\begin{aligned}
E[q(p)] & =z_{A}(p) \int_{Q_{0}(p) / z_{A}(p)}^{u} x f(x) d x \\
& =\Gamma(p) \\
& :=\frac{(a-b p) u^{2}}{2(u-l)}-\frac{g^{2}}{2(u-l)(p-h)^{2}(a-b p)} .
\end{aligned}
$$

The first and second derivatives of $E[q(p)]$ w.r.t. $p$ are given by the below, respectively:

$$
\begin{aligned}
& \frac{d \Gamma(p)}{d p}=\frac{\left[g^{2}(2 a+b h-3 b p)-b u^{2}(p-h)^{3}(a-b p)^{2}\right]}{2(u-l)(p-h)^{3}(a-b p)^{2}} \\
& \frac{d^{2} \Gamma(p)}{d p^{2}}= \\
& \quad \times\left[18 b^{2}\left(p-\frac{(2 a+b h)}{3 b}\right)^{2}+(a-b h)^{2}\right] \\
& <0 \quad \forall p>0 .
\end{aligned}
$$

Therefore, $\Gamma(p)$ is strictly concave in $p$ for all $p>0$. Hence, there is a unique maximum of $\Gamma(p)$. Solving the first-order condition by (A.21), the unique optimal $p$ that maximizes $\Gamma(p)$ is given by

$$
\widehat{p}_{4 A}=\underset{p}{\arg }\left\{g^{2}(2 a+b h-3 b p)-b u^{2}(p-h)^{3}(a-b p)^{2}=0\right\} .
$$

If $p_{L, 3 A}<\widehat{p}_{4 A}<p_{H, 3 A}$, then $p=\widehat{p}_{4 A}$ is the optimal solution of maximizing $E[q(p)]$, and the corresponding optimal MPQ is $Q_{4 A}{ }^{*}=Q_{0}\left(\widehat{p}_{4}\right)=g /\left(\widehat{p}_{4}-h\right)$.

Next, from the proof of Proposition 7 , $\lim _{p \rightarrow p_{H, 3 A}} Q_{0}(p) / z_{A}(p)=\lim _{p \rightarrow p_{L, 3 A}} Q_{0}(p) / z_{A}(p)=u$, or equivalently, $\lim _{p \rightarrow p_{H, 3 A}} \eta(p)=\lim _{p \rightarrow p_{L, 3 A}} \eta(p)=0$ $\left(\eta(p)\right.$ is given by (A.16)). Therefore, $\lim _{p \rightarrow p_{H, 3 A}} E[q(p)]=$ $\lim _{p \rightarrow p_{L, 3 A}} E[q(p)]=0$. If $\widehat{p}_{4 A} \leq p_{L, 3 A}$ or $\widehat{p}_{4 A} \geq p_{H, 3 A}$, then by the strict concavity of $\Gamma(p), \Gamma(p)<0$ for all $p_{L, 3 A}<p<p_{H, 3 A}$. By definition, $E[q(p)] \geq 0$. Therefore, $E[q(p)] \neq \Gamma(p)$ and $\left(Q_{0}(p), p\right)$ are not feasible for all $p>0$ in this case.

Proof of Proposition 8. Let $K_{1 M}(Q)=Q[h+g / Q]^{b} / a$.

(a) From Propositions 1 and 2 , for any $Q>0$, the designer will set the retail price as $p_{1 M}{ }^{*}(Q)=p_{0}(Q)=h+g / Q$. Next, for $\left(Q, p_{0}(Q)\right)$ being feasible, as $l<x<u$, we need to have $K_{1 M}(Q)<u$. Moreover, we have $d K_{1 M}(Q) / d Q=$ $[h+g / Q]^{b-1}\{h+g(1-b) / Q\} / a$.

(b) The expected profit of the retailer is given by

$$
\begin{aligned}
& E\left[\pi_{1 M}(Q)\right] \\
& =\int_{K_{1 M}(Q)}^{u}\left(\left[p_{1 M}{ }^{*}(Q)-h\right] x z_{M}\left[p_{1 M}{ }^{*}(Q)\right]-g\right) f(x) d x \\
& =\frac{g\left[u-K_{1 M}(Q)\right]}{(u-l)}\left[\frac{a}{Q}\left(\frac{g}{Q}+h\right)^{-b}\left(\frac{u+K_{1 M}(Q)}{2}\right)-1\right] .
\end{aligned}
$$


The first derivative of $E\left[\pi_{1 M}(Q)\right]$ is

$$
\begin{aligned}
& \frac{d E\left[\pi_{1 M}(Q)\right]}{d Q} \\
& \quad=-a g Q^{-3}\left[h+\frac{g}{Q}\right]^{-b-1}\left[u^{2}-K_{1 m}^{2}(Q)\right] \frac{h Q-(b-1) g}{2(u-l)} .
\end{aligned}
$$

For $0<b \leq 1$, we have $h Q-(b-1) g \geq h Q>0$; so $d E\left[\pi_{1 M}(Q)\right] / d Q<0$ for all $Q$. Therefore, $E\left[\pi_{1 M}(Q)\right]$ is strictly decreasing in $Q$ and the optimal MPS should be the smallest possible value of $Q$. For $Q=0, K_{1 M}(0)=0$ (as $\left.K_{1 M}(Q)=\left\{Q^{1 / b} h+g Q^{1 / b-1}\right]\right\}^{b} / a$ and $\left.0<b \leq 1\right)$, and hence $E\left[\pi_{1 M}(0)\right]=+\infty$.

(c) For $b>1, d E\left[\pi_{1 M}(Q)\right] / d Q>0$ for $Q<(b-$ 1) $g / h, d E\left[\pi_{1 M}(Q)\right] / d Q=0$ for $Q=(b-1) g / h$, and $d E\left[\pi_{1 M}(Q)\right] / d Q<0$ for $Q>(b-1) g / h$. Therefore, $E\left[\pi_{1 M}(Q)\right]$ is unimodal for $Q>0$.

(c-i) $d K_{1 M}(Q) / d Q<0$ for all $Q<g(b-1) / h$; $d K_{1 M}(Q) / d Q=0$ for all $Q=g(b-1) / h$; and $d K_{1 M}(Q) / d Q>$ 0 for all $Q>g(b-1) / h$. Therefore, $K_{1 M}(Q)$ is minimized at $Q=g(b-1) / h$. As $K_{1 M}(g(b-1) / h)=g(b-1)[h b /(b-1)]^{b} / a h$, if $g(b-1)[h b /(b-1)]^{b} / a h \geq u$, then the Retailer's participation constraint is not satisfied for any $Q>0$; that is, $K_{1 M}(Q)>u$ for all $Q>0$.

(c-ii) If $g(b-1)[h b /(b-1)]^{b} / a h<u$, the optimal MPQ is uniquely given by $Q_{1 M}{ }^{*}=(b-1) g / h$, and by direct manipulation, the optimal retail price is uniquely given by $p_{1 M}{ }^{*}=p_{1 M}{ }^{*}\left(Q_{1 M}{ }^{*}\right)=b h /(b-1)$.

Proof of Proposition 9. (a) Under the "Project-oriented Dled" scenario with $z(p)=z_{M}(p)=a p^{-b}$, by Proposition 3 , the optimal MPQ is $Q_{0}(p)=g /(p-h)$, which satisfies (C1). Since $l \leq x \leq u$, to ensure $\left(Q_{3 M}{ }^{*}(p), p\right)$ being feasible, we need to have $Q_{0}(p) / z_{M}(p)<u$, which is equivalent to

$$
K_{3 M}(p):=\frac{g p^{b}}{a(p-h)}<u .
$$

(b) and (c) We have $d K_{3 M}(p) / d p=\left(g p^{b} / a(p-h)^{2}\right)[(b-1) p-$ $b h]$. Next, anticipating $Q_{0}(p)$, the DPS feasibility probability is given by

$$
\Psi_{3 M}(p)=1-F\left[\frac{Q_{0}(p)}{z_{M}(p)}\right] .
$$

If $l<K_{3 M}(p)<u$,

$$
\Psi_{3 M}(p)=-\frac{g}{a(u-l)}\left(\frac{p^{b}}{p-h}\right)+\left(1+\frac{l}{u-l}\right) ;
$$

and the first derivative of $\Psi_{3 M}(p)$ w.r.t. $p$ is

$$
\frac{d \Psi_{3 M}(p)}{d p}=-\frac{g p^{b-1}}{a(u-l)(p-h)^{2}}[(b-1) p-h b] .
$$

If $l \geq K_{3 M}(p), \Psi_{3 M}(p)=1$; and if $u \leq K_{3 M}(p), \Psi_{3 M}(p)=0$ (note that $\left(Q_{3 M}{ }^{*}(p), p\right)$ is not feasible in this case, so it can be ignored in the rest of the proof).
For $b \leq 1$, as $d K_{3 M}(p) / d p<0$ for all $p>0$, there exist unique $p_{3 M l}{ }^{\prime}$ and unique $p_{3 M u}{ }^{\prime}$, such that (i) $p_{3 M l}{ }^{\prime}>p_{3 M u}{ }^{\prime}>$ $h$, (ii) $K_{3 M}\left(p_{3 M l}{ }^{\prime}\right)=l$, and (iii) $K_{3 M}\left(p_{3 M u}{ }^{\prime}\right)=u$. Observed from (A.29) that $d \Psi_{3 M}(p) / d p>0$ for all $p>0$ satisfying $l<K_{3 M}(p)<u$. Therefore, $\Psi_{3 M}(p)$ is strictly increasing in $p$ for all $p_{3 M u}{ }^{\prime}<p<p_{3 M l}{ }^{\prime}$, and $\Psi_{3 M}(l)=1$ for all $p \geq p_{3 M l}{ }^{\prime}$ $\left(\left(Q_{0}(p), p\right)\right.$ is not feasible for any $\left.p<p_{3 M u}{ }^{\prime}\right)$.

For $b>1, d K_{3 M}(p) / d p<0$ for all $h<p<h b /(b-1)$; $d K_{3 M}(p) / d p=0$ for $p=h b /(b-1)$; and $d K_{3 M}(p) / d p>0$ for all $p>h b /(b-1)$. Thus, $-K_{3 M}(p)$ is unimodal in $p$ for $p>h$, and is maximized at $p=\widehat{p}_{3 M}:=h b /(b-1)$. As $K_{3 M}\left(\widehat{p}_{3 M}\right)=$ $(g(b-1) / a h)(h b /(b-1))^{b}$, if $(g(b-1) / a h)(h b /(b-1))^{b} \geq u$, then $K_{3 M}(p) \geq u$ for all $p>h$. Therefore, the pair $\left(Q_{0}(p), p\right)$ is feasible if and only if $(g(b-1) / a h)(h b /(b-1))^{b}<u$.

For $b>1$ and $(g(b-1) / a h)(h b /(b-1))^{b}<u$, as $K_{3 M}(h)=+\infty$, there exist $p_{3 M u}$ " and $p_{3 M u}{ }^{\prime \prime \prime}$ such that (i) $h<p_{3 M u}{ }^{\prime \prime}<p_{3 M u}{ }^{\prime \prime \prime}$, (ii) $K_{3 M}\left(p_{3 M u^{\prime \prime}}{ }^{\prime \prime}=K_{3 M}\left(p_{3 M u}{ }^{\prime \prime \prime}\right)=u\right.$, and (iii) $K_{3 M}(p)<u$ for all $p_{3 M u}{ }^{\prime \prime}<p<p_{3 M u}{ }^{\prime \prime \prime}$. If $(g(b-1) / a h)(h b /(b-1))^{b}>l$, then $l<K_{3 M}(p)<u$ for all $p_{3 M u}{ }^{\prime \prime}<p<p_{3 M u}{ }^{\prime \prime \prime}$. Otherwise, there exist $p_{3 M l}{ }^{\prime \prime}$ and $p_{3 M l}{ }^{\prime \prime \prime}$, such that (i) $p_{3 M u}{ }^{\prime \prime}<p_{3 M l}{ }^{\prime \prime} \leq p_{3 M l}{ }^{\prime \prime \prime}<p_{3 M u}{ }^{\prime \prime \prime}$, (ii) $K_{3 M}\left(p_{3 M l}{ }^{\prime \prime}\right)=K_{3 M}\left(p_{3 M l}{ }^{\prime \prime \prime}\right)=l$, (iii) $l<K_{3 M}(p)<u$ for all $p_{3 M u}{ }^{\prime \prime}<p<p_{3 M l}$, or $p_{3 M l}{ }^{\prime \prime \prime}<p<p_{3 M u}$ '"' , and (iv) $K_{3 M}(p) \leq l$ for all $p_{3 M l}<p<p_{3 M l}$ '"'

Next, for $b>1$ and $l<K_{3 M}(p)<u, d \Psi_{3 M}(p) / d p>0$ for all $h<p<h b /(b-1) ; d \Psi_{3 M}(p) / d p=0$ for $p=h b /(b-1)$; and $d \Psi_{3 M}(p) / d p<0$ for all $p>h b /(b-1)$.

Combining the above findings, for $b>1$ and $(g(b-$ 1) $/ a h)(h b /(b-1))^{b}<u$, if $(g(b-1) / a h)(h b /(b-1))^{b}>l$, then $\Psi_{3 M}(p)$ is unimodal in $p$ and is maximized at $p=$ $h b /(b-1)$. Otherwise, $\Psi_{3 M}(p)$ is strictly increasing in $p$ for all $p_{3 M u}{ }^{\prime \prime}<p^{\prime \prime}<p_{3 M l} l^{\prime \prime}$, is strictly decreasing in $p$ for all $p_{3 M l}{ }^{\prime \prime \prime}<$ $p<p_{3 M u}{ }^{\prime \prime \prime}$, and $\Psi_{3 M}(p)=1$ for all $p_{3 M l}{ }^{\prime \prime}<p<p_{3 M l}{ }^{\prime \prime \prime}$.

Proof of Proposition 10. By Proposition 3(a), anticipating $Q_{0}(p)=g /(p-h)$, the expected sales volume is given by

$$
\begin{aligned}
E\left[q_{4 M}(p)\right] & =\Gamma_{1}(p):=\int_{K_{3 M}(p)}^{u} x f(x) d x \\
& =\frac{u^{2}}{2(u-l)}-\frac{g^{2} p^{2 b}}{2 a^{2}(u-l)(p-h)^{2}},
\end{aligned}
$$

for $l<K_{3 M}(p)<u$, where $K_{3 M}(p)$ is given by (23),

$$
\begin{gathered}
E\left[q_{4 M}(p)\right]=\frac{u-l}{2}, \quad \text { for } K_{3 M}(p) \leq l \\
E\left[q_{4 M}(p)\right]=0, \quad \text { for } K_{3 M}(p) \geq u
\end{gathered}
$$

The first and second derivatives of $\Gamma_{1}(p)$ wr.t. $p$ are derived below, respectively:

$$
\frac{d \Gamma_{1}(p)}{d p}=-\frac{g^{2} p^{2 b-1}}{a^{2}(u-l)(p-h)^{3}}[(b-1) p-b h] .
$$

For $b \leq 1$, observing from (A.32), $d \Gamma_{1}(p) / d p>0$ for all $p>0$. Therefore, $E\left[q_{4 M}(p)\right]$ is strictly increasing in $p$ for all $l<K_{3 M}(p)<u$. From the proof of Proposition 8, 
$d K_{3 M}(p) / d p<0$ for all $p>0$, for $b \leq 1$. Thus, $l<K_{3 M}(p)<$ $u$ implies $p_{3 M u}{ }^{\prime}<p<p_{3 M l}{ }^{\prime}$, and $K_{3 M}(p) \leq l$ implies $p \geq$ $p_{3 M l}{ }^{\prime}$, where $K_{3 M}\left(p_{3 M l}{ }^{\prime}\right)=l$ and $K_{3 M}\left(p_{3 M u}{ }^{\prime}\right)=u$ (see the proof of Proposition 8 for details). Moreover, $E\left[q_{4 M}(p)\right]<$ $E\left[q_{4 M}\left(p_{3 M l}{ }^{\prime}\right)\right]=(u-l) / 2$ for all $p_{3 M u}{ }^{\prime}<p<p_{3 M l}{ }^{\prime}$, and $E\left[q_{4 M}(p)\right]=(u-l) / 2$ for all $p \geq p_{3 M l}$.

For $b>1$ and $l<K_{3 M}(p)<u, d \Gamma_{1}(p) / d p>0$ for all $h<p<h b /(b-1) ; d \Gamma_{1}(p) / d p=0$ for $p=h b /(b-1)$; and $d \Gamma_{1}(p) / d p<0$ for all $p>h b /(b-1)$. Next, from the proof of Proposition 8:

(i) for $b>1$, the pair $\left(Q_{0}^{*}(p), p\right)$ is feasible if and only if $(g(b-1) / a h)(h b /(b-1))^{b}<u ;$

(ii) for $b>1$ and $(g(b-1) / a h)(h b /(b-1))^{b}<u$, $K_{3 M}(p)<u$ for all $p_{3 M u}{ }^{\prime \prime}<p<p_{3 M u}{ }^{\prime \prime}$;

(iii) for $b>1$ and $l<(g(b-1) / a h)(h b /(b-1))^{b}<u$, $l<K_{3 M}(p)<u$ for all $p_{3 M u}{ }^{\prime \prime}<p<p_{3 M u}{ }^{\prime \prime \prime}$ and

(iv) for $b>1$ and $(g(b-1) / a h)(h b /(b-1))^{b} \leq l, l<$ $K_{3 M}(p)<u$ for all $p_{3 M u}{ }^{\prime \prime}<p<p_{3 M l}{ }^{\prime \prime}$ or $p_{3 M l}{ }^{\prime \prime \prime}<$ $p<p_{3 M u}{ }^{\prime \prime \prime}$, and $K_{3 M}(p) \leq l$ for all $p_{3 M l}{ }^{\prime \prime}<p<$ $p_{3 M l}$.

Combining the above findings, for $b>1$ and $(g(b-$ $1) / a h)(h b /(b-1))^{b}<u$, if $(g(b-1) / a h)(h b /(b-1))^{b}>l$, then $E\left[q_{4 M}(p)\right]$ is unimodal in $p$ and is maximized at $p=$ $h b /(b-1)$. Otherwise, $E\left[q_{4 M}(p)\right]$ is strictly increasing in $p$ for all $p_{3 M u}{ }^{\prime \prime}<p<p_{3 M l}$ ", is strictly decreasing in $p$ for all $p_{3 M l}{ }^{\prime \prime \prime}<p<p_{3 M u}{ }^{\prime \prime \prime}$, and $E\left[q_{4 M}(p)\right]=(u-l) / 2$ for all $p_{3 M l}{ }^{\prime \prime}<p<p_{3 M l}{ }^{\prime \prime \prime}$.

\section{Conflict of Interests}

The authors declare that there is no conflict of interests regarding the publication of this paper.

\section{Acknowledgment}

This paper is partially supported by the "985 Project" of Sun Yat-Sen University. The authors sincerely thank the anonymous reviewers for their constructive comments on this paper.

\section{References}

[1] M. Newbery, A Study of the UK Designer Fashion Sector, Department of Trade \& Industry and the British Fashion Council, London, UK, 2003.

[2] J. Richardson, "Vertical integration and rapid response in fashion apparel," Organization Science, vol. 7, no. 4, pp. 400-412, 1996.

[3] C. Mills, "Enterprise orientations: a framework for making sense of fashion sector start-up," International Journal of Entrepreneurial Behaviour and Research, vol. 17, no. 3, pp. 245271, 2011.

[4] J. Howe, "The rise of crowdsourcing," Wired Magazine, vol. 14, no. 6, pp. 1-4, 2006.

[5] K. J. Boudreau, N. Lacetera, and K. R. Lakhani, "Incentives and problem uncertainty in innovation contests: an empirical analysis," Management Science, vol. 57, no. 5, pp. 843-863, 2011.
[6] P. M. Di, M. M. Wasko, and R. E. Hooker, "Getting customers' ideas to work for you: learning from dell how to succeed with online user innovation communities," MIS Quarterly Executive, vol. 9, no. 4, pp. 213-228, 2010.

[7] Y. Huang, P. V. Singh, and T. Mukhopadhyay, "How to design crowdsourcing contest: a structural empirical analysis," Working Paper, 2012.

[8] B. L. Bayus, "Crowdsourcing new product ideas over time: an analysis of the Dell IdeaStorm community," Management Science, vol. 59, no. 1, pp. 226-244, 2013.

[9] Y. Wang, "Joint pricing-production decisions in supply chains of complementary products with uncertain demand," Operations Research, vol. 54, no. 6, pp. 1110-1127, 2006.

[10] A. J. Dalal and M. Alghalith, "Production decisions under joint price and production uncertainty," European Journal of Operational Research, vol. 197, no. 1, pp. 84-92, 2009.

[11] B. Jiang, K. Jerath, and K. Srinivasan, "Firm strategies in the "mid tail" of platform-based retailing," Marketing Science, vol. 30, no. 5, pp. 757-775, 2011.

[12] Y. M. Li and Y. L. Lee, "Pricing peer-produced services: quality, capacity, and competition issues," European Journal of Operational Research, vol. 207, no. 3, pp. 1658-1668, 2010.

[13] P. Li-Liang and T. Jiang, "Study on pricing level of C2C electronic commerce platform," in Proceedings of the International Conference on Management Science and Engineering (ICMSE '11), pp. 452-455, Rome, Italy, September 2011.

[14] K. S. Anand and R. Aron, "Group buying on the web: a comparison of price-discovery mechanisms," Management Science, vol. 49, no. 11, pp. 1546-1562, 2003.

[15] B. A. Pasternack, "Optimal pricing and return policies for perishable commodities," Marketing Science, vol. 4, no. 2, pp. 166-176, 1985.

[16] G. Cai, Z. G. Zhang, and M. Zhang, "Game theoretical perspectives on dual-channel supply chain competition with price discounts and pricing schemes," International Journal of Production Economics, vol. 117, no. 1, pp. 80-96, 2009.

[17] N. C. Petruzzi and M. Dada, "Pricing and the newsvendor problem: a review with extensions," Operations Research, vol. 47, no. 2, pp. 183-194, 1999.

[18] C. T. Yang, L. Y. Ouyang, and H. H. Wu, "Retailer's optimal pricing and ordering policies for non-instantaneous deteriorating items with price-dependent demand and partial backlogging," Mathematical Problems in Engineering, vol. 2009, Article ID 198305, 18 pages, 2009.

[19] H. Chen, Y. Chen, C. H. Chiu, T. M. Choi, and S. Sethi, "Coordination mechanism for the supply chain with leadtime consideration and price-dependent demand," European Journal of Operational Research, vol. 203, no. 1, pp. 70-80, 2010.

[20] C. H. Chiu, T. M. Choi, and C. S. Tang, "Price, rebate, and returns supply contracts for coordinating supply chains with price-dependent demands," Production and Operations Management, vol. 20, no. 1, pp. 81-91, 2011.

[21] M. Fisher and A. Raman, "Reducing the cost of demand uncertainty through accurate response to early sales," Operations Research, vol. 44, no. 1, pp. 87-99, 1996.

[22] S. C. Graves, A. H. G. R. Kan, and P. H. Zipkin, Handbooks in Operations Research and Management Science, vol. 4 of Logistics of Production and Inventory, North Holland, Amsterdam, The Netherlands, 1993. 
[23] P. S. Chow, T. M. Choi, and T. C. E. Cheng, "Impacts of minimum order quantity on a quick response supply chain," IEEE Transactions on Systems, Man, and Cybernetics A, vol. 42, no. 4, pp. 868-879, 2012. 


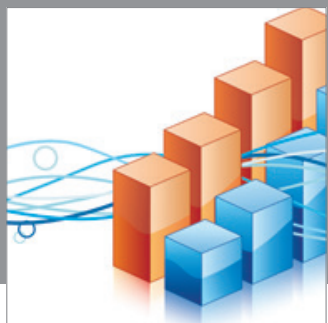

Advances in

Operations Research

mansans

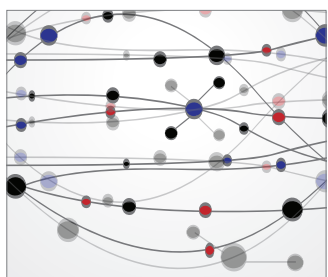

The Scientific World Journal
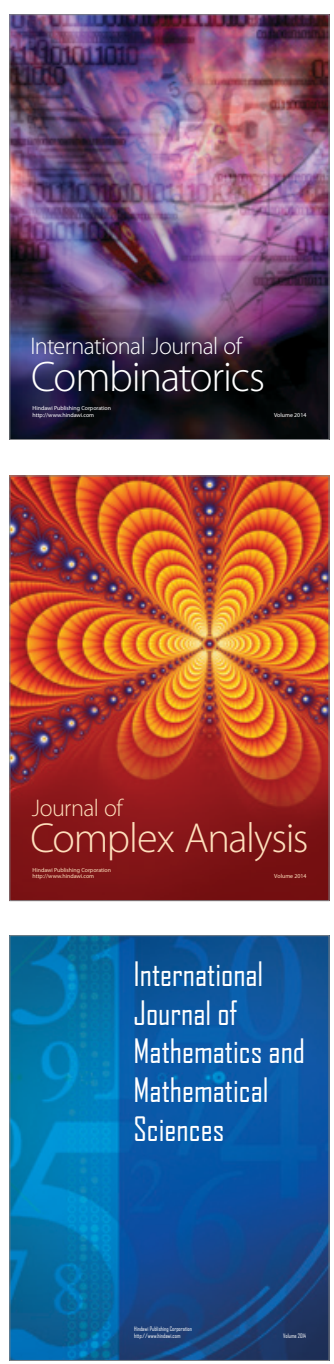
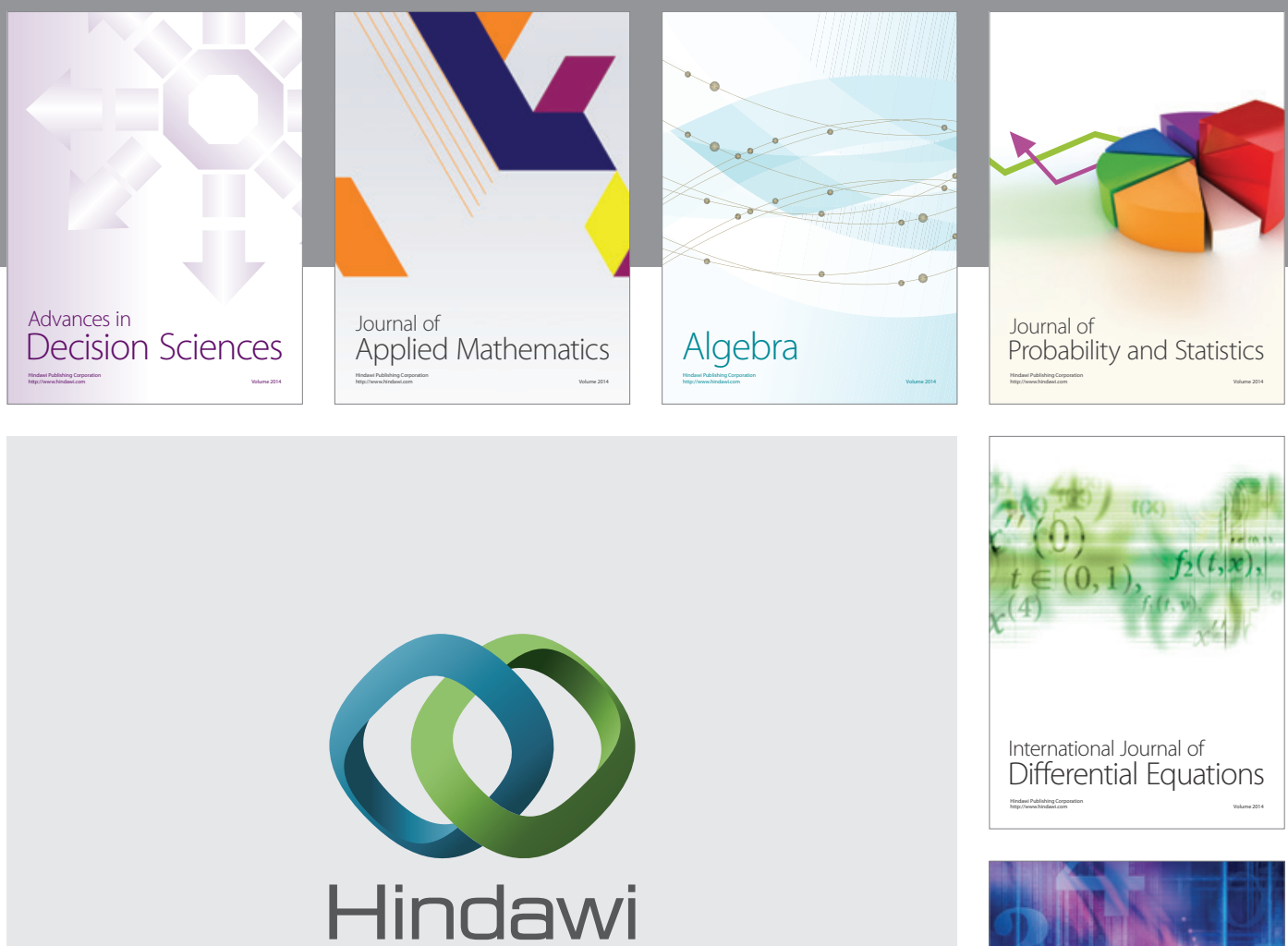

Submit your manuscripts at http://www.hindawi.com
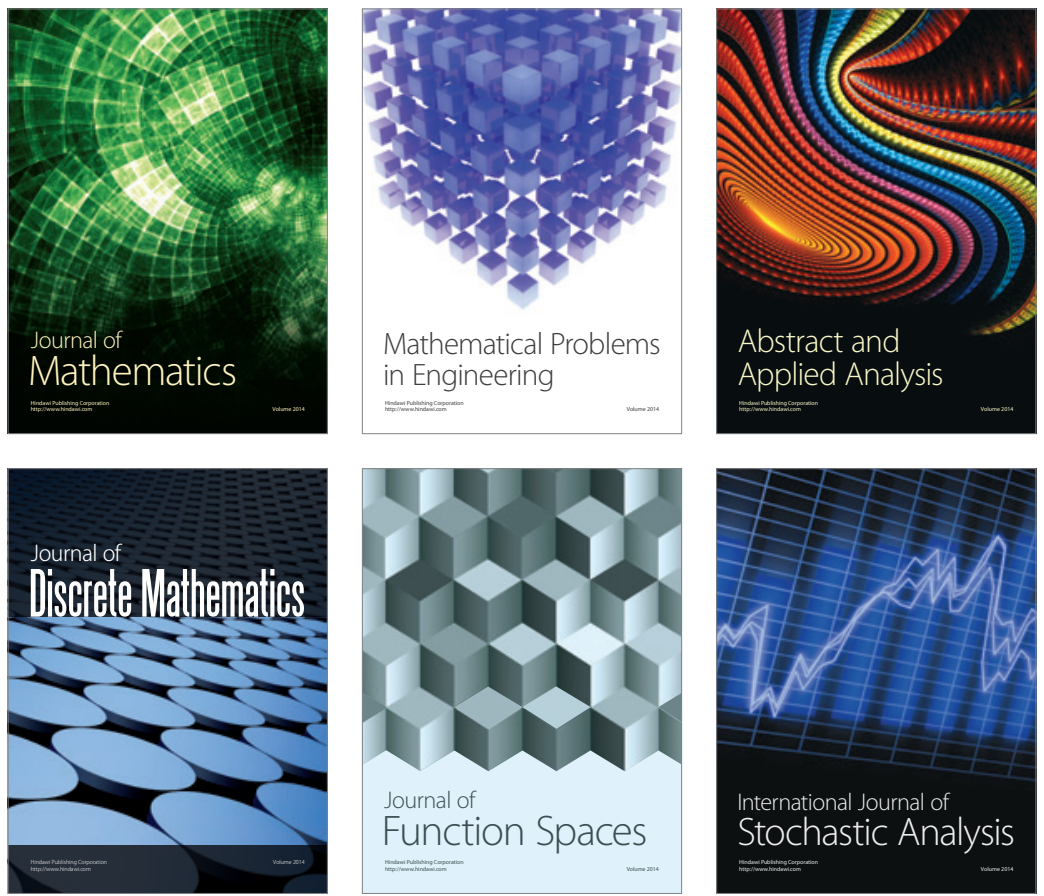

Journal of

Function Spaces

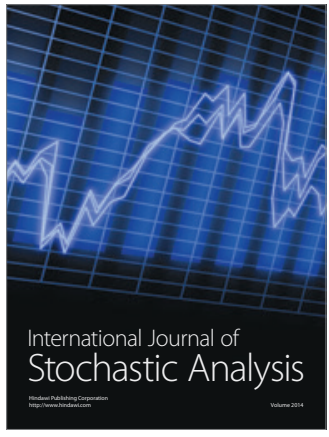

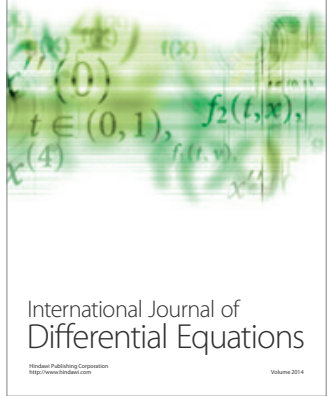
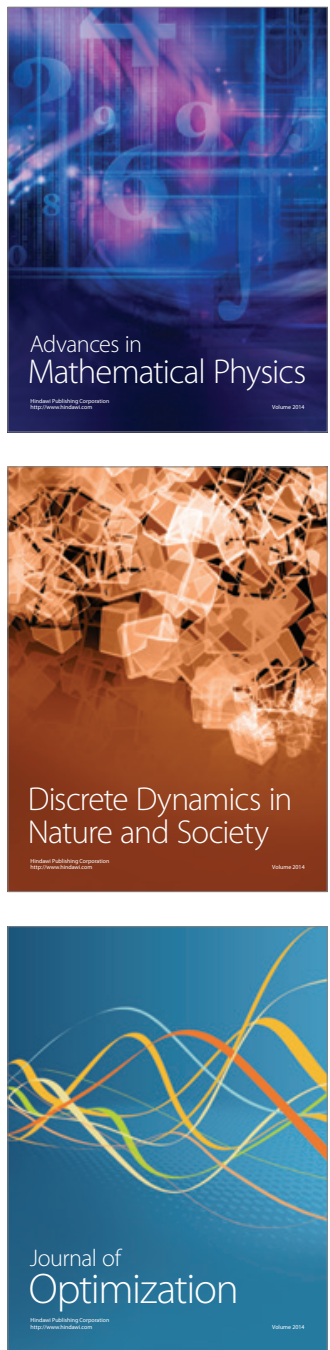